\title{
Neuer Fragebogen zur Qualität der Weiterbildung
}

\author{
Max Giger, Leiter Ressort Medical Education FMH, Winterthur
}

Aus den Resultaten der bisherigen Umfragen konnten nur wenig konkrete Massnahmen abgeleitet werden. Dieses Jahr wird ein neuer Fragebogen zum Einsatz kommen, der mit allen Betroffenen entwickelt werden soll und einen Testlauf zu bestehen hat.
Um den verschiedenen Kritiken Rechnung zu tragen, beschloss der Zentralvorstand FMH, die Umfrage bei den Assistentinnen und Assistenten in neuem Gewand mit zielgerichteten Fragen durchzuführen. Die Fragen sollen in denjenigen Gebieten gestellt werden, in welchen die Befragten und die Beurteilten die Möglichkeit zu Reaktionen auf die Antworten sehen.

Mit der Durchführung des Projektes «Neuer Fragebogen» wurde das Institut für Sozialpsychologie I der Universität Zürich beauftragt. Um die interessierenden Fragefelder zu erfahren, fand im Januar 2003 je ein Workshop mit Leiterinnen und Leitern von Weiterbildungsstätten bzw. mit Assistentinnen und Assistenten statt. Die Anonymität der Antwortenden wird aus Gründen des Persönlichkeitsschutzes wohl weiterhin gewährt bleiben müssen. Der neue Fragebogen soll benutzerfreundlich bzw. innerhalb weniger als einer Viertelstunde zu beantworten sein. Auch sollen falsche Angaben durch entsprechende Rückfragen aufgedeckt werden. Die definitive Auswahl der Fragen wird nach einem Testlauf im Mai erfolgen. Der neue Fragebogen soll im August 2003 versandt werden.

An der Entstehung und Ausgestaltung des neuen Fragebogens sollen möglichst alle Betroffenen beteiligt werden. Das Forum «Neuer Fragebogen zur Weiterbildung» auf der Homepage der FMH (www.fmh/awf) soll allen diese Möglichkeit bieten. Fragen und Bemerkungen sind erwünscht, um das Projekt zu unterstützen. dungsstätten äusserten. Von seiten der Leiter der Weiterbildungsstätten wurde wiederholt moniert, dass sie aus den Antworten keine konkreten Schlüsse ziehen können. 